\title{
Sustainable construction for a circular economy
}

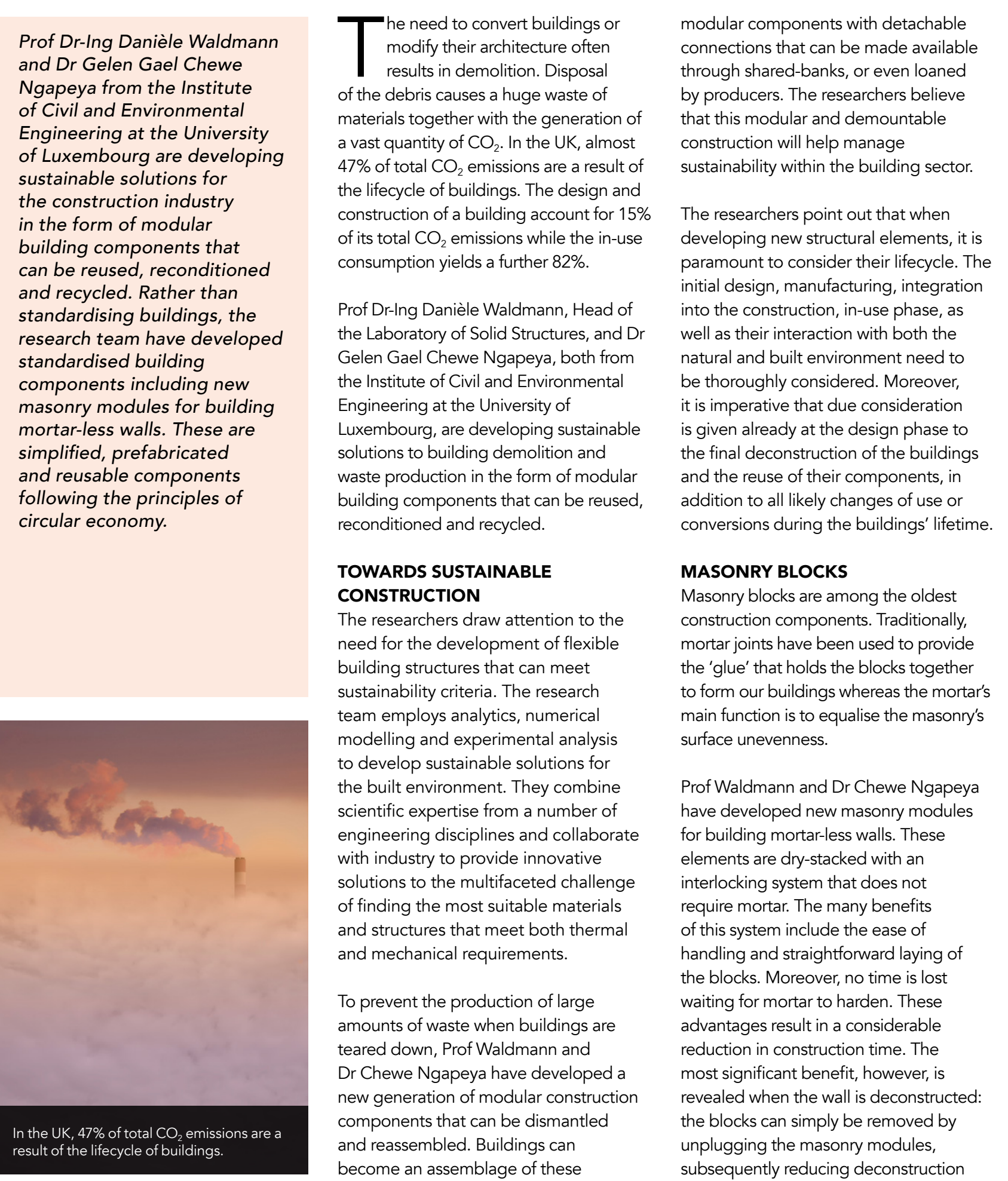

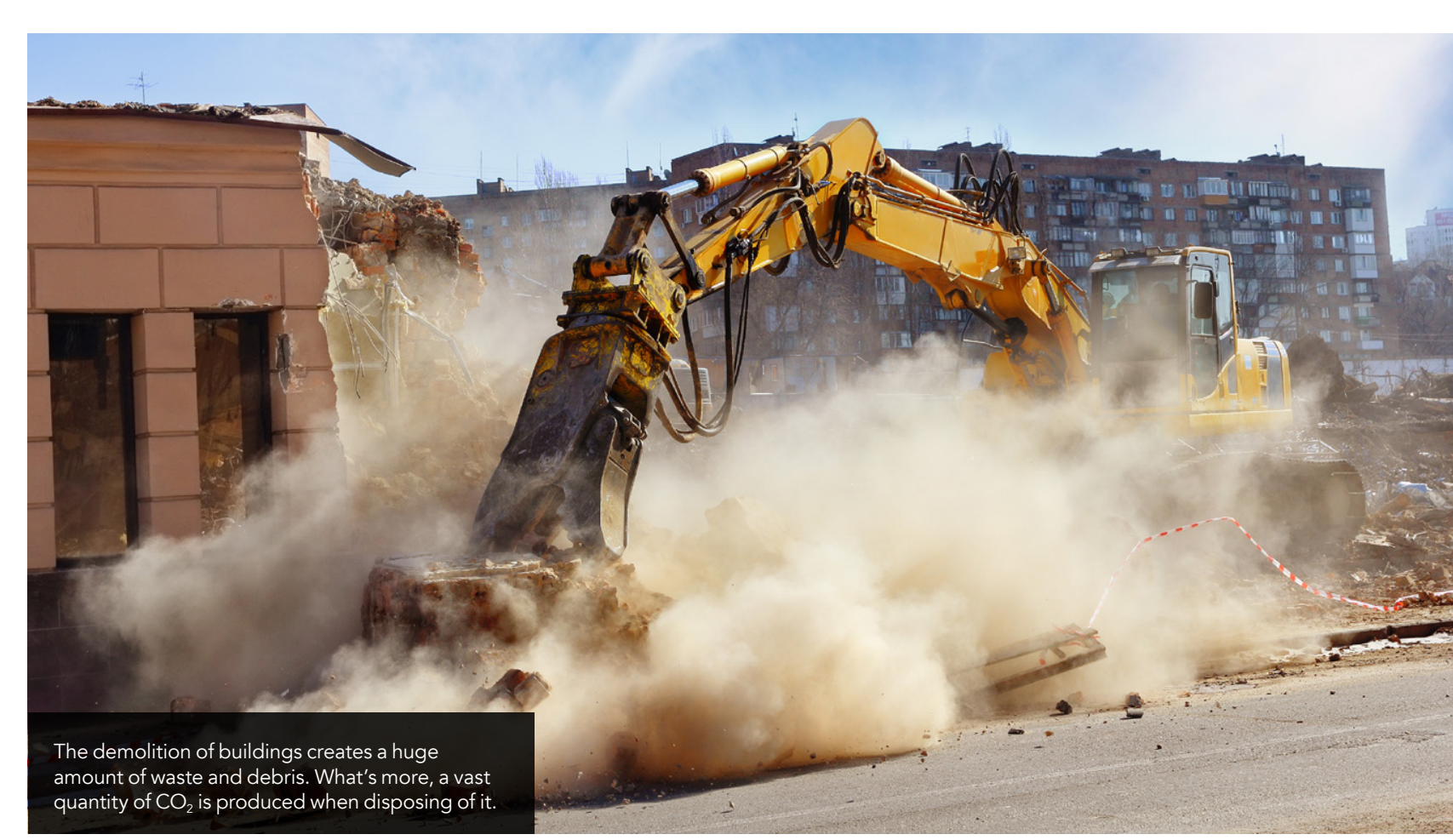
time. Furthermore, the modular units can have developed a design method for predicting the load-bearing capacity of peaks induced by the blocks' geometric imperfections: the height difference and the surface roughness. The researchers For addressing the impact of the

To prevent large amounts of waste when buildings are teared down, the team has developed modular construction components that can be dismantled and reassembled.

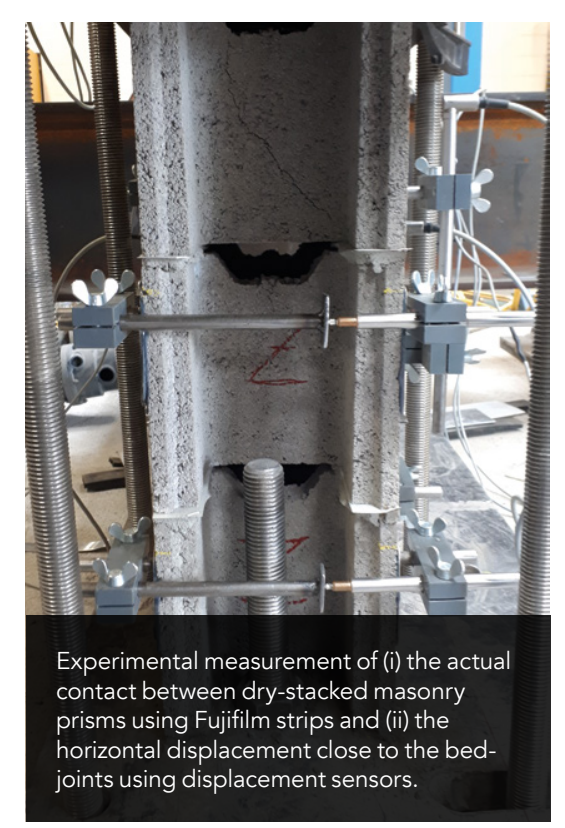

the block performance in compression ests were carried out to examine the Large-scale walls were built and subjected to innovative tests in collaboration with a ocal industrial partner. The methodology involved placing Fuji films into the horizontal layers in order to investigate the real contact areas of the intermediate interfaces during the static loading tests. Experimental tests were carried out on 2.5-metre-high walls which were put weighing up to 180 tonnes.

\section{PARAMETRIC STUDIES}

Non-linear finite element calculations have so been completed. The key scientific of a new simulation technique that mimis the distribution and intensity of the load on the wall using a selection of heightto-length ratios. This analytical algorithm revealed that the changes in bearing capacity and stress distribution in the mortar-less walls were functions of the wall height and length, as well as the height variation of masonry block units.

Research has shown that the materia used to make the block, the way of loading, the failure mechanism as well as imperfections in the bed joints influence the compression strengths and
the behaviour of dry-stacked masonry. contact properties of the intermediate 


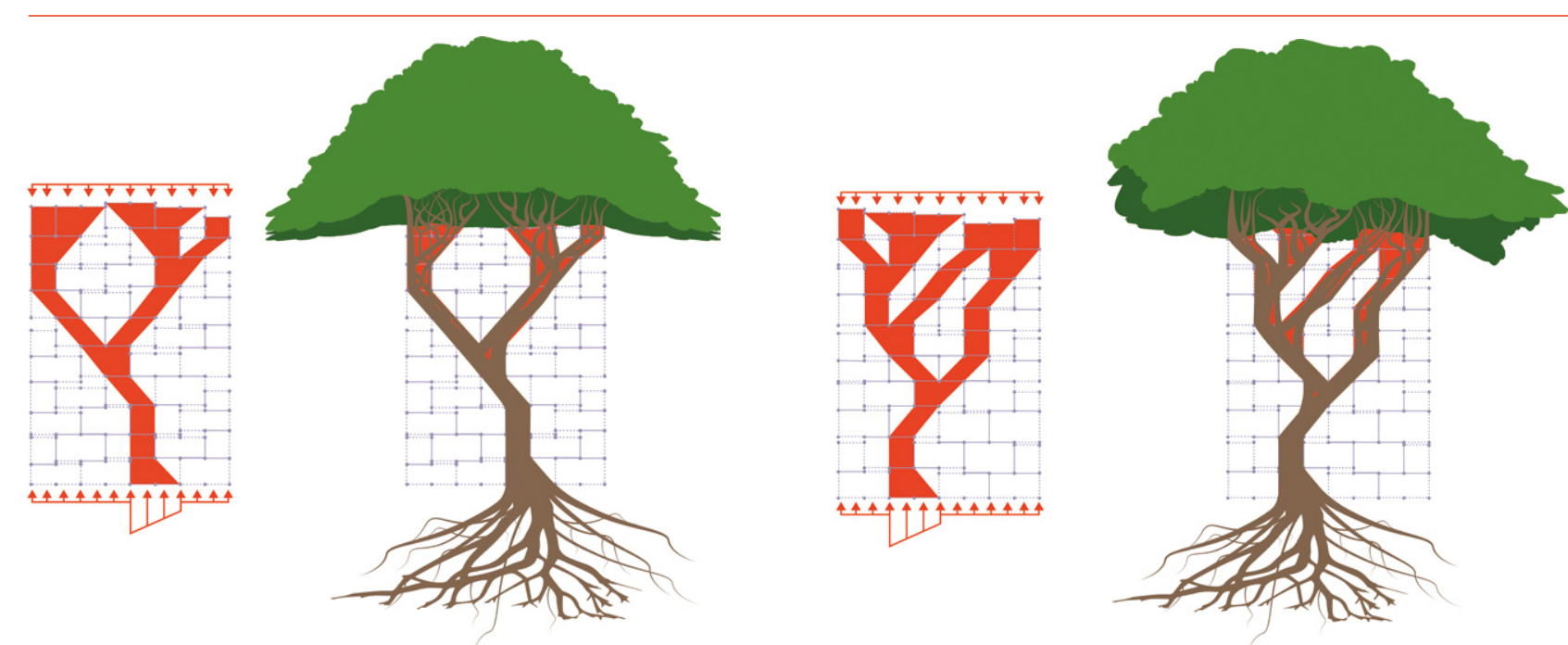

The stress distribution (red) is related to the height and surface imperfection of the blocks. From the top to the bottom of the walls, the stresses percolate
where there is a contact between the masonry blocks, which gives the 'tree-like' load percolation system

The variation in block height dictates the rate of contact between courses, therefore determining the load perculation system within the wall.

Solid blocks exhibit higher compressive strength than hollow ones. The two kinds of bed joint imperfections regularly observed in dry-stacked masonry are the roughness of the bed joints and the variation in height of the blocks. These uneven joints and variation in block height educe the contact surface area between the courses. This results in uneven load transfer when a uniform compressive load applied to the top course of dry-stacked masonit blocks is cunevenly transferred to he course below. This can result in stress concentration thatredur

\section{BED JOINT ROUGHNESS}

If the block's shape permits it,

grinding in order to reduce the bed joint imperfections, but this extra process slows production, adds to the unit cost and still leaves a slight roughness profile on the surface. Most blocks, therefore, do not undergo this process. It has been shown that bed joint imperfections in dry-stacked masonry can result up to $50 \%$ of reduction in load-bearing capacity when compared with mortared masonry blocks. Previous to the current research, there have been few studes, however, hro inproving the compressive strength of dry-stacked bed joint roughness.
The researchers have investigated applying a contact layer to the top face of dry-stacked masonry blocks during manufacture to level the unpredictable roughness of the bed joints. This bi-layer design should increase both the contact area and the loadbearing capacity of dry-stacked masonry. They trialled three different materials as contact layers: a mineral layer mortar with lightweight aggregate, an external mineral render both of medium stiffiness and an auxetic material of low stiffness. Auxe materials have a negative Poisson's stretched, they become thicker in the direction perpendicular to the applied force. These materials tend to have high energy absorption and fracture resistance properties. The team found that rather than acting as a bonding agent, the auxetic material significantly reduced the lateral expansion near the bed joints, therefore increasing the loadbearing capacity

\section{HEIGHT IMPERFECTIONS}

Traditionally, the mortar joints between courses absorb the variation in the heigh of the blocks. Dry-stacked masonry block cannot avail of this correction and the of contact between courses, therefor variation in block height dictates the rate system within the wall. The researchers investigated the impact of geometric load bering hapaty masony walls. They have develaced an algorithm to calculate the stress amplification in a wall based on block height imperfection. This innovative computational input than the existing finite element method.

\section{SUSTAINABLE CONSTRUCTION}

The research team at the Laboratory of

Solid Structures are continuing to explore optimised design concepts for structura elements of green buildings with the analytical and experimental analysis pionstaced the development of the

The environmental and economic goals have prompted the research group to focus on eco-construction, also known as the circular economy: looking beyond the traditional make, use, dispose mode and aiming to design out waste and pollution by keeping resources in use for as long as possible. They endeavour to extract the maximum possible value from resources during their service life and then recover and regenerate products and materials from them at the end of their lifespan. Prof Waldmann and Dr research projects will be developed collaboration with strategic research and industrial partners to promote analytical approach requires much less further development of the numerical, sustainable construction, together with. Chewe Ngapeya conclude that "further

\section{Behind the Research}

\section{Prof Danièle Waldmann-Diederich}

E: daniele.waldmann@uni.lu W: Wttps://wwwen.uni.lu/research/stm/doe/members/daniele_waldmann

\section{Research Objectives}

Prof Dr Waldmann and Dr Chewe Ngapeya research among others how modular components help drive sustainability in the building sector.

\section{Detail}

Professor Danièle Waldmann-Diederich 6 avenue de la Fonte Luxembourg

Professor Danièle Waldmann-Diederich joined the University of Luxembourg in 2003. She established the "Solid Structures (LSS)" research group, which innovated in (1) Structural Health Monitoring, (2) Reliable structural nalysis of reinforced or prestressed concrete structures, (3) Inventive concrete formulations by substitutions (3)ing waste or renewable products and (4) Advanced Numerical Simulation (since 2018)

Funding

tzebuerger CONTERN
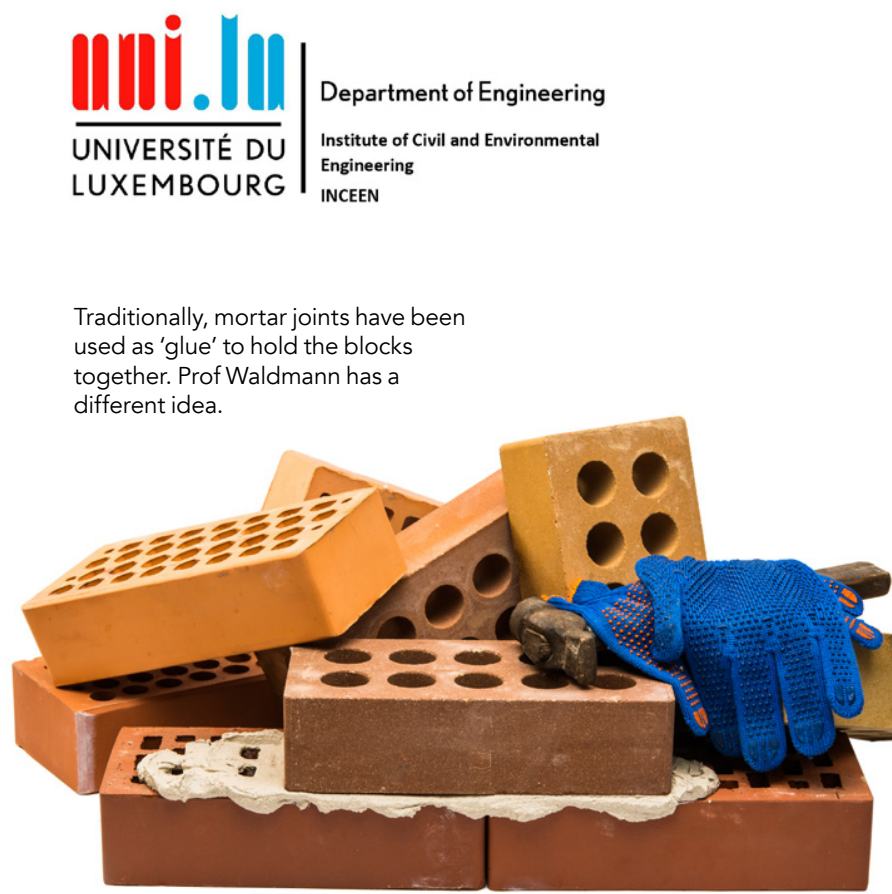

References

Chewe Ngapeya, G.G., and Waldmann, D. (2020) Experimental and analytical analysis of the load-bearing capacity $\mathrm{P}_{\mathrm{u}}$ of improved dry-stacked masonry. Journal of Building Engineering, [online] 27. Available at: https://doi org/10.1016/j.jobe.2019.100927

Chewe Ngapeya, G.G., and Waldmann, D. (2020). Overcome of bed-joint imperfections and improvement of actual contact in dry-stacked masony, Colstre th hand Bulling Matenials, conbuildmat.2019.117173

Chewe Ngapeya, G.G., Waldmann, D., and Scholzen, F. (2018). Impact of the height imperfections of masonry blocks on the load bearing capacity of dry-stack masonry walls. Construction and Building Materials, 165, 898-913. Available at: https://doi.org/10.1016/j.conbuildmat.2017.12.183

Waldmann, D., and Poulles, R. (2016). Research for sustainable construction. Science and Technology, 20.

Agaajani, S, Waldmann, D., Scholzen, F., and Louge, A. (2016). Numerical analysis for the determination of stress percolation in dry-stacked wall systems. Masonry International, 29, 27-38.

Agaajani, S. (2015). Development and Investigation of a New Dry-stacked Wall System, University of Luxembourg, Thesis.

\section{Personal Response}

What sparked your interest in developing reusable

\section{building components?}

Nowadays, human environment is facing challenges induced by pollution and accumulation of inert industry. This initiated main paradigm shift torruction demountable construction in general Indeed, reusable building components exhibit various advantages: they are timesaving, waste the least amount of materials, are easy to construct and deconstruct, and have the Our interest was sparked by throughout their life cycle. art research on the design methods of demountable construction components in line with new policy (CEAP) of the European Union. 